\title{
A aplicação de polímeros biodegradáveis como uma alternativa sustentável
}

\author{
The application of biodegradable polymers as a sustainable alternative \\ La aplicación de polímeros biodegradables como alternativa sostenible
}

Recebido: 14/07/2021 | Revisado: 21/07/2021 | Aceito: 23/07/2021 | Publicado: 31/07/2021

\author{
Bruna Aline Araújo \\ ORCID: https://orcid.org/0000-0002-9429-6994 \\ Universidade Federal de Campina Grande, Brasil \\ E-mail: brunaaaraujo15@gmail.com \\ Lucas Silva de Freitas \\ ORCID: https://orcid.org/0000-0002-1329-9457 \\ Universidade Federal do Recôncavo da Bahia, Brasil \\ E-mail: lucasfreitas852@gmail.com \\ Kênia Kelly Freitas Sarmento \\ ORCID: https://orcid.org/0000-0001-6815-9577 \\ Universidade Estadual da Paraíba, Brasil \\ E-mail: keniakellys41@gmail.com \\ Vanessa Rosales Bezerra \\ ORCID: https://orcid.org/0000-0002-7920-4107 \\ Universidade Estadual da Paraíba, Brasil \\ E-mail: rosalesuepb@gmail.com \\ Carlos Antônio Pereira de Lima \\ ORCID: https://orcid.org/0000-0002-1301-6066 \\ Universidade Estadual da Paraíba, Brasil \\ E-mail: caplima@uepb.edu.br \\ Keila Machado de Medeiros \\ ORCID: https://orcid.org/0000-0001-9250-1432 \\ Universidade Federal do Recôncavo da Bahia, Brasil \\ E-mail:keilamedeiros@ufrb.edu.br
}

\begin{abstract}
Resumo
A geração de resíduos poliméricos apresentam três aspectos: o volume crescente, a complexidade e a poluição ambiental. A reciclagem vem com o intuito de diminuir a quantidade destes resíduos gerados, consistindo em um processo de transformação de materiais, previamente separados, de forma a possibilitar a sua recuperação. $\mathrm{Na}$ atual conjuntura, a degradação biológica por meio dos polímeros biodegradáveis é considerada a nova tendência sustentável. Os polímeros biodegradáveis se comparados com os polímeros sintéticos se degradam com menos tempo pela ação de microrganismos, possuindo propriedades semelhantes entre si. Este trabalho tem como objetivo descrever sobre a degradação biológica de materiais poliméricos, levando em consideração os polímeros biodegradáveis naturais e sintéticos. De maneira geral, os polímeros biodegradáveis naturais possuem uma alta taxa de renovabilidade e podem ser utilizados tanto na área médica, como na área ambiental, pois após o descarte são biodegradados por microrganismos presentes no meio ambiente, em um curto espaço de tempo se comparados com os materiais poliméricos tradicionais. Os polímeros biodegradáveis sintéticos têm sido mais largamente empregados em aplicações biomédicos por apresentarem excelente resistência mecânica, bioabsorvível, biocompatibilidade, biodegradabilidade, não toxicidade, flexibilidade e, além disso, são aplicados em embalagens especiais.
\end{abstract}

Palavras-chave: Degradação biológica; Biopolímeros; Polímeros biodegradáveis; Sustentabilidade.

\begin{abstract}
The generation of polymeric waste has three aspects: the increasing volume, complexity and environmental pollution. Recycling comes with the aim of reducing the amount of these residues generated, consisting of a process of transformation of materials, previously separated, in order to allow their recovery. In the current situation, biological degradation through biodegradable polymers is considered the new sustainable trend. Biodegradable polymers compared to synthetic polymers degrade in less time due to the action of microorganisms, having properties similar to each other. This work aims to describe the biological degradation of polymeric materials, taking into account natural and synthetic biodegradable polymers. In general, natural biodegradable polymers have a high rate of renewability and can be used both in medical and environmental areas, since after disposal they are biodegraded by microorganisms present in the environment, in a short time compared with traditional polymeric materials. Synthetic biodegradable polymers have been more widely used in biomedical applications because they present excellent mechanical resistance, bioabsorbable, biocompatibility, biodegradability, non-toxicity, flexibility and, moreover, are applied in special packaging.
\end{abstract}

Keywords: Biological degradation; Biopolymers; Biodegradable polymers; Sustainability. 


\begin{abstract}
Resumen
La generación de residuos poliméricos tiene tres aspectos: aumento de volumen, complejidad y contaminación ambiental. El reciclaje nace con el objetivo de reducir la cantidad de estos residuos generados, consistente en un proceso de transformación de materiales, previamente separados, para posibilitar su valorización. En la situación actual, la degradación biológica a través de polímeros biodegradables se considera la nueva tendencia sostenible. Los polímeros biodegradables, en comparación con los polímeros sintéticos, se degradan en menos tiempo debido a la acción de los microorganismos, teniendo propiedades similares entre sí. Este trabajo tiene como objetivo describir la degradación biológica de materiales poliméricos, teniendo en cuenta los polímeros biodegradables naturales y sintéticos. En general, los polímeros biodegradables naturales tienen una alta tasa de renovabilidad y pueden usarse tanto en el área médica como ambiental, ya que después de su eliminación son biodegradables por microorganismos presentes en el ambiente, en un corto período de tiempo en comparación con los materiales poliméricos tradicionales. Los polímeros sintéticos biodegradables han sido más utilizados en aplicaciones biomédicas debido a su excelente resistencia mecánica, bioabsorbibilidad, biocompatibilidad, biodegradabilidad, no toxicidad, flexibilidad y, además, se aplican en envases especiales.
\end{abstract}

Palabras clave: Degradación biológica; Biopolímeros; Polímeros biodegradables; Sostenibilidad.

\title{
1. Introdução
}

O descarte de materiais polímeros por indústrias e a população em geral, gera o acúmulo de resíduos que pode passar pelo processo de reciclagem, com o objetivo de fundir o polímero e produzir um novo produto com as mesmas características ou diferentes. Há uma contribuição para o meio ambiente quando se recicla polímeros e se compra polímeros reciclados, pois muitos destes são descartados em locais proibidos, lagos e rios, evitando a poluição do meio ambiente, além de se produzir menos polímeros devido a sua reciclagem (Ashton et al., 2016).

A reciclagem de polímeros consiste em um processo de transformação, previamente separados, de forma a possibilitar a sua recuperação. Esses materiais podem ter duas origens, no caso de rejeitos de processo industrial ou de produto pósconsumo. Os resíduos são recuperados por meios de operações que permitem que materiais já processados sejam aproveitados como matéria-prima no processo gerador ou em outros processos (Dias, 2016).

Segundo a Sociedade Americana de Ensaios e Materiais (ASTM), os tipos de reciclagem de polímeros englobam a divisão reciclagem primária, reciclagem secundária, reciclagem terciária e reciclagem quaternária (Fraga, 2014). Na reciclagem primária, a matéria-prima é de fonte absolutamente confiável e limpa, como no caso, de resíduos da indústria de polímeros. O processo utilizado normalmente envolve a seleção dos resíduos, moagem (após o material adquirir o formato de floco), secagem e reprocessamento em equipamentos como extrusoras e injetoras. Nos filmes polímeros, pode haver uma etapa extra de aglutinação para aumentar a densidade dos flocos e torná-los mais aptos ao reprocessamento. O produto final é o material reciclado com propriedades semelhantes à resina virgem (Piva \& Wiebeck, 2004).

Quando o produto final possui características, desempenho e propriedades mecânicas inferiores às comparadas com o produto obtido com a resina virgem, a reciclagem é definida como secundárias e se aplica normalmente a resíduos pósconsumo (Hatti-Kaul et al., 2019). Na reciclagem secundária, a matéria-prima é proveniente de resíduos sólidos e o processo se baseia em seleção, moagem, lavagem, secagem e reprocessamento, incluindo aglutinação no caso de filmes. A matériaprima da reciclagem secundária pode ser eventualmente de resíduo industrial, porém, por definição, o produto final é um material reciclado com propriedades inferiores a resina virgem (Piva \& Wiebeck, 2004).

Na reciclagem terciária é quando o processo utilizado para reciclar o polímero é baseado na despolimerização, ou seja, no nível químico, é promovida a decomposição química controlada do material, tendo como produtos, oligômeros, monômeros e substâncias de baixa massa molar, que posteriormente poderão ser submetidos a novos processos de polimerização, processamento, industrialização e utilização (Valerio, Muthuraj \& Codou, 2020).

$\mathrm{Na}$ reciclagem quaternária é quando o processo de reciclar o plástico é baseado na sua combustão, visando o aproveitamento do seu conteúdo energético. Os produtos finais são a energia e a emissão gasosa, notadamente dióxido de 
carbono $\left(\mathrm{CO}_{2}\right)$, quando ocorre a combustão completa. De acordo com a ASTM, devido à necessidade em se mencionar o processo, difundiu-se outra classificação, que divide a reciclagem de polímeros em reciclagem mecânica, reciclagem química e reciclagem energética (Thomas et al., 2020).

A reciclagem mecânica é quando o polímero passa por etapas de seleção, moagem, lavagem, secagem, aglutinação e reprocessamento, dando origem ao grânulo ou uma peça polimérica reciclada. Pode envolver aditivação do polímero, visando à melhoria de suas propriedades finais. As propriedades do reciclado pode ou não ser semelhantes às da resina virgem, isso dependerá de uma série de fatores, como qualidade de cada etapa do processo e da matéria-prima (resíduo) utilizada (Hamad, Kaseem e Deri, 2013).

A reciclagem mecânica é a mais difundida, pois não necessita de muita tecnologia para implementá-la. A reciclagem mecânica é mais empregada para termoplásticos, mas também poderá ser empregada para borrachas, dependendo da sua composição. Atualmente, a reciclagem mecânica parece ser a opção mais promissora do ponto de vista econômico e ambiental (Maris et al., 2018).

A reciclagem química é quando o polímero passa pela despolimerização, visando à destruição da estrutura polimérica, inclusive da cadeia principal. Já a reciclagem energética é quando o polímero passa pela combustão. O polímero não deixa de ser despolimerizado, porém não há ênfase nesses produtos, e sim na energia desprendida no processo total (Braido, Borges \& Pinto, 2018; Valerio, Muthuraj \& Codou, 2020).

A reciclagem energética consiste na compactação dos resíduos e subsequente queima, gerando energia, resíduos sólidos e gasosos. Este processo é baseado no alto poder calorífico dos polímeros, utilizando-os como combustíveis sintéticos. Os resíduos gasosos passam inicialmente por filtros ou precipitadores eletrostáticos para a remoção de material particulado e as partículas mais finas existentes no gás (Piva \& Wiebeck, 2004). Posteriormente, esses resíduos gasosos passam por lavadores de gases, para a remoção dos gases ácidos à base de enxofre e nitrogênio por meio de pulverização de água, resultando em um gás limpo, gerando um efluente líquido de ácido com material particulado, que tem maior facilidade de tratamento (Gomes, 2010; Vesilind \& Morgan, 2011).

A reciclagem energética também é conhecida como pirólise, quando a decomposição térmica é realizada em ausência de oxigênio e temperaturas controladas $\left(760\right.$ a $\left.1400^{\circ} \mathrm{C}\right)$, é chamada de incineração quando a fragmentação térmica é realizada por oxidação. A pirólise tem como produto resíduo gasoso e o óleo de pirólise. Já a incineração têm resíduos sólidos (cinzas), resíduos gasosos e energia (Mano, Pacheco \& Bonelli, 2010). Esta reciclagem é utilizada para resíduos complexos (termorrígidos, borrachas e compósitos) ou que necessitem de cuidados especiais como descartáveis médico-hospitalares, embalagens de óleos, entre outros. Os resíduos gasosos são tratados de forma a reduzir a toxicidade dessas emissões, os sólidos (subproduto da queima) recebem destinação diferenciadas e adequadas, conforme o país que utiliza este processo e a energia térmica gerada é recuperada sob a forma de vapor ou eletricidade.

A degradação biológica é uma alternativa para a reciclagem convencional (mecânica, química e energética) e para o desenvolvimento sustentável, por ser uma degradação biológica, utilizando organismos vivos encontrados no solo. A biodegradação pode ser definida como a degradação de um material orgânico causada por atividade biológica, principalmente pela ação enzimática de microorganismos. Existem alguns métodos de avaliação da biodegradação dos polímeros como, monitorar o crescimento de microorganismos, o consumo do substrato (polímero), a liberação de $\mathrm{CO}_{2}$ e mudanças nas propriedades do polímero (Brito et al., 2011).

A compostagem é um processo que controla a decomposição biológica e transformação de materiais biodegradáveis. Os polímeros compostáveis são aqueles que sofrem biodegradação durante a compostagem para ceder $\mathrm{CO}_{2}$, água, compostos inorgânicos e biomassa. Existem polímeros chamados de polímeros oxo-biodegradáveis, que consistem em polímeros contendo aditivos que aceleram sua degradação oxidativa na presença de luz ou calor (Brito et al., 2011). 
A biodeterioração de materiais é a biodegradação de diferentes materiais por diferentes tipos de microrganismos, é um processo em que os microrganismos atacam e colonizam as superfícies poliméricas na forma de biofilmes, os quais são uma mistura de microrganismos, água, polissacarídeos e proteínas que, em contato com o polímero sintético, causam algumas modificações, é um processo que depende das condições do meio, dos tipos de microrganismos e da estrutura do polímero (Franchetti e Marconato, 2006).

Os biopolímeros são materiais poliméricos classificados estruturalmente como polissacarídeos, poliésteres ou poliamidas. A matéria-prima para sua manufatura é uma fonte de carbono renovável, geralmente um carboidrato derivado de plantios comerciais de larga escala como cana-de-açúcar, milho, batata, trigo e beterraba; ou um óleo vegetal extraído de soja, girassol, palma ou outra planta oleaginosa (Pradella, 2006).

Há grandes impactos ambientais causados pelos processos de extração e refino utilizados para produção dos polímeros provenientes do petróleo, a escassez do petróleo e o aumento do seu preço são alguns fatores que estão diretamente relacionados ao crescente interesse pelos biopolímeros (Brito et al., 2011).

Os polímeros biodegradáveis são polímeros que podem ser degradados pela ação de microrganismos, como bactérias e fungos. O interesse nesses polímeros tem crescido nos últimos anos, uma vez que os mesmos têm sido considerados uma alternativa aos polímeros convencionais, como por exemplo, na agricultura. A biodegradação é uma solução muito promissora, uma vez que é menos agressiva ao meio ambiente, além de completar o ciclo de carbono e do nitrogênio. E quando o polímero biodegradável é submetido à compostagem, obtém-se um material rico em carbono e que pode retornar ao solo com efeitos benéficos (Rosa \& Pantano Filho, 2003).

Alternativas têm sido buscadas com o objetivo de substituir os materiais poliméricos convencionais que sejam mais compatíveis com a preservação ambiental. Assim, um vasto número de polímeros biodegradáveis tem sido sintetizado recentemente e alguns microrganismos e enzimas capazes de degradá-los têm sido identificados (Smith, 2005).

O potencial para a produção de polímeros biodegradáveis de baixo custo, utilizando sistemas de produção renovável (milho, mandioca, feijão, soja) estimulam grandes empresas a investir cada vez mais nesse mercado competitivo (Lendlein \& Sisson, 2011). Diante do exposto, este trabalho tem como objetivo descrever sobre a degradação biológica de materiais poliméricos, considerando as novas tendências sustentáveis que são os polímeros biodegradáveis naturais e sintéticos.

\section{Revisão Bibliográfica}

\subsection{Biodegradação}

A degradação de plástico sintético é muito lenta e pode levar até 500 anos. A degradação destes polímeros gera resíduos menores, que apesar de não ser evidente, se acumulam nos ecossistemas em grandes quantidades (Farias et al., 2016). A biodegradação consiste na decomposição do polímero pela ação de enzimas produzidas por microrganismos, tais como fungos e bactérias (Boonmee, 2016). A principal forma de ataque enzimático nos polímeros é através de reações oxidativas, o que causa o rompimento da cadeia principal, e uma redução na massa molar (Fechini, 2013). O processo de degradação biológica ocorre na presença de oxigênio, nutrientes, umidade, temperatura e potencial hidrogeniônico $(\mathrm{pH})$ adequado.

A biodegradação é um processo natural em que microorganismos (bactéria e fungo) e suas enzimas utilizam os compostos orgânicos como fonte de alimentos, convertendo em composto mais simples que estão redistribuídos por ciclo elementares como o do carbono, nitrogênio e enxofre. Quando entram em contato com os polímeros, os microrganismos secretam enzimas que conseguem transformar as macromoléculas em segmentos menores, permitindo que estes sejam consumidos. Em geral, derivam deste processo $\mathrm{CO}_{2}, \mathrm{CH}_{4}$, componentes microbianos e outros produtos, sendo assim, a biodegradação é um processo biótico, necessitando de seres vivos para ocorrer (Fechini, 2013). 
Sob condições apropriadas de umidade, temperatura, ph, disponibilidade de oxigênio e presença de material nutriente, a biodegradação é um processo relativamente rápido. Um tempo de biodegradação de 2 a 3 anos no máximo é um parâmetro razoável para a completa assimilação e o desaparecimento de um material plástico. Contudo, novas normas técnicas sobre biodegradabilidade foram apresentadas para decomposição e mineralização (Fechini, 2013). O processo químico da biodegradação de polímeros sob condições aeróbicas e anaeróbicas (Cana) podem ser vistos pelas equações 1 e 2 :

$$
\begin{aligned}
& \mathrm{C}_{\text {aeróbicas: }} \mathrm{C}_{\text {polímero }}+\mathrm{O}_{2}=>\mathrm{CO}_{2}+\mathrm{H}_{2} \mathrm{O}+\mathrm{C}_{\text {resíduo }} \\
& \mathrm{C}_{\text {anaeróbicas: }} \mathrm{C}_{\text {polímero }}=>\mathrm{CO}_{2}+\mathrm{CH}_{4}+\mathrm{H}_{2} \mathrm{O}+\mathrm{C}_{\text {resíduo }}
\end{aligned}
$$

Tanto os fungos como as bactérias produzem enzimas que hidrolisam os substratos para que sejam utilizados como materiais nutrientes. Os fungos são os microrganismos responsáveis pela biodegradação de polímeros de origem natural, como o amido e a celulose, e sua ação sempre ocorre em ambiente aeróbico. Já as bactérias agem tanto em ambientes aeróbicos como anaeróbicos (Fechini, 2013). A biodegradação do polímero é um processo que consiste na modificação física ou química, causada pela ação de microrganismos, sob certas condições de calor, umidade, luz, oxigênio e nutrientes orgânicos e minerais adequados (Franchetti e Marconato, 2006). Os fatores que influenciam na biodegradação do polímero são a sua estrutura química, a morfologia do polímero, a exposição a alguns tipos de radiação e a massa molar (Fechini, 2013).

\subsection{Biopolímeros}

Os biopolímeros são polímeros feitos a partir de fontes renováveis como resíduos de milho, cana de açúcar entre outros resíduos. Os biopolímeros possuem as mesmas propriedades do plástico comum, celulose, amido, quitina, proteínas, são exemplos de biopolímeros. Os biopolímeros de origem natural têm atraído atenção significativa nos últimos anos devido à sua abundância e propriedades versáteis como não toxicidade, biocompatibilidade, biodegradabilidade, flexibilidade e renovabilidade (Bilal \& Iqbal, 2019). A produção global de biopolímeros em 2017 foi de cerca de 2,05 milhões de toneladas e prevê-se que os biopolímeros atinjam 2,44 milhões de toneladas em 2022. Apesar do mercado dinâmico, os biopolímeros representam menos de 1\% da produção total de polímeros que foi de cerca de 320 milhões de toneladas em 2017 (Niaounakis, 2019). A Figura 1 ilustra o número de pesquisas que desenvolveram materiais alternativos ao ambiente.

Figura 1: Publicações anuais sobre biopolímeros até 2015.

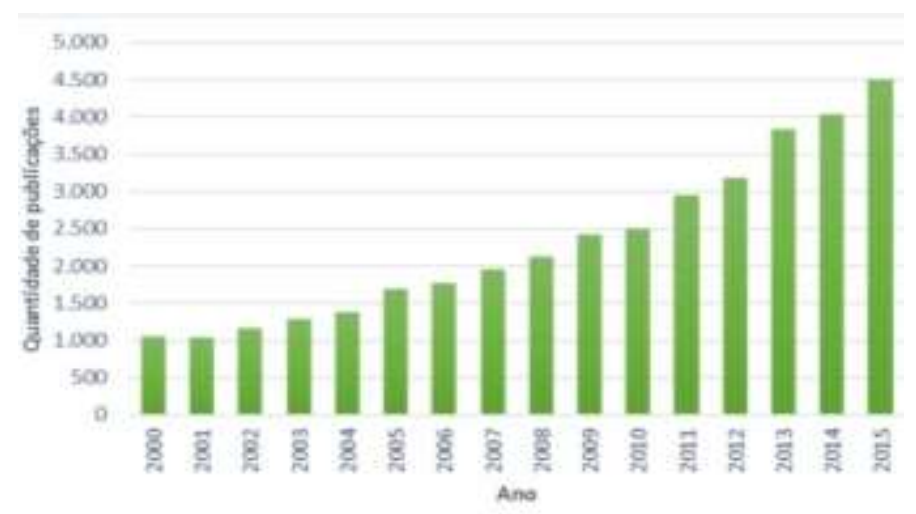

Fonte: Farias et al. (2016).

A Tabela 1 apresenta a possibilidade de substituição de polímeros de fontes fósseis por biopolímeros, que são o amido, o poli ácido lático (PLA), o polihidroxibutirato (PHB), e o polihidroxibutirato-copolihdroxihexanoato (PHBHx). 
Tabela 1: Potencial de substituição de polímeros vindos de fontes fósseis por biopolímeros.

\begin{tabular}{l|c|c|c|c|c|c|c|c|c}
\hline Polimero & PVC & PEAD & PEBD & PP & PS & PMMA & PA & PET & PC \\
\hline Amido & - & + & + & + & + & - & - & - & - \\
PLA & - & + & - & + & + & - & + & + & - \\
PHB & - & + & - & ++ & + & - & - & - & - \\
PHBHx & + & ++ & + & ++ & + & - & - & + & - \\
\hline
\end{tabular}

Fonte: Brito et al. (2011).

Os biopolímeros possuem algumas limitações técnicas que tornam difícil sua processabilidade, então devido estas limitações técnicas, blendas, compósitos e nanocompósitos têm sido estudados no intuito de melhorar as seguintes propriedades: processabilidade, resistência térmica, propriedades mecânicas e propriedades reológicas (Brito et al., 2011).

Bilal e Iqbal (2019) estudaram os biopolímeros de origem natural, como alginato, quitosana e celulose, os esforços de pesquisa têm se concentrado em biopolímeros naturais como novos materiais de suporte/compósitos para diversas aplicações nos setores biomédico, ambiental, farmacêutico, de alimentos e na indústria de biocombustíveis/energia. Este trabalho destaca o desenvolvimento recente e o uso de biopolímeros e seus compostos avançados como portadores de suporte para a imobilização de uma variedade de enzimas diferentes para desenvolver biocatalisadores. De acordo com os resultados, foi possível constatar que a exploração de biopolímeros como materiais de suporte para múltiplas aplicações é um campo emergente que oferece potencial inexplorado para buscar inovações.

Niaounakis (2019) investigou as principais tecnologias de reciclagem de biopolímeros, incluindo biocombustíveis e/ou polímeros biodegradáveis, divulgados na literatura de patentes. As principais tecnologias de reciclagem examinadas foram: reciclagem mecânica e reciclagem química. Portanto, o autor concluiu que o processamento mecânico de resíduos pósindustriais pode ser a única opção realista para a reciclagem do PLA biodegradável e que o politetrafluoretileno (PTFE) pode ser misturado com polietileno tereftalato (PET) industrial em quantidade de até $2 \%$ e ser reciclado.

\subsection{Polímeros Biodegradáveis}

Os polímeros biodegradáveis são polímeros que se degradam com menos tempo do que os polímeros sintéticos, possuindo propriedades semelhantes entre si, eles se degradam pela ação de microorganismos de ocorrência natural como bactérias, fungos e algas, podendo ser consumidos em semanas ou meses sob condições favoráveis de biodegradação. Os polímeros biodegradáveis podem ser derivados de fontes naturais renováveis como, por exemplo, o milho, celulose, batata e cana de açúcar, ou serem sintetizados por bactérias a partir de pequenas moléculas como o ácido butírico ou o ácido valérico dando origem ao PHB e ao polihidroxibutirato-co-valerato (PHB-HV), respectivamente, ou até mesmo serem derivados de fonte animal, como a quitina, a quitosana ou proteínas. Os polímeros biodegradáveis também podem ser obtidos de fontes fósseis, petróleo, ou da mistura entre biomassa e petróleo. Os polímeros biodegradáveis do petróleo mais conhecidos são as policaprolactonas (PCL), as poliesteramidas, os copoliésteres alifáticos e os copoliésteres aromáticos (Brito et al., 2011).

Algumas vantagens dos polímeros biodegradáveis seriam a produção em escala industrial, pois o Brasil é um grande produtor de milho, batata e cana de açúcar. Estes polímeros possuem boa resistência mecânica e baixa toxicidade, o tempo de degradação é outra vantagem, pois é mais rápido em comparação com os polímeros tradicionais (Fechini, 2013).

A degradação de um polímero é um processo ocasionado por vários fatores responsáveis pela perda de algumas de suas propriedades físicas nesses processos em geral ocorre cisão da cadeia polimérica e também a quebra de estrutura no retículo cristalino. Os fatores que podem provocar a degradação de um polímero podem ser decorrentes do processamento e dos efeitos ambientais sobre os mesmos (Lima \& Okimoto, 2009). 
Certas características dos polímeros podem influenciar o processo de degradação. Além da estrutura química dos polímeros, há outros fatores que também influenciam a velocidade de degradação. Na celulose a alta percentagem de cristalinidade determina uma velocidade da degradação menor se comparada a do amido que apresenta baixa cristalinidade. Polímeros amorfos tendem a degradar mais rapidamente, pelo menor empacotamento das cadeias (Fechini, 2013).

Os polímeros biodegradáveis mostram um campo em desenvolvimento, com crescente utilização destes tipos de polímeros não só para embalagens, bem como para diversos setores, se tornando uma área de grande potencial de estudos para viabilização do seu uso. No entanto, dificuldades devem ser superadas, para uma maior utilização destes polímeros, que no Brasil ainda é baixo, além do seu custo, quando comparado aos das resinas convencionais (Landim et al., 2016).

\section{Metodologia}

Para a produção deste estudo, uma revisão bibliográfica qualitativa, buscou realizar uma pesquisa sistemática nas bases de dados SciELO, Sciencedirect e Web of Science, que contemplasse artigos de períodicos com o assunto de interesse, de preferência por artigos da última década. Os resultados da pesquisa foram obtidos durante todo o primeiro semestre de 2021, sendo as palavras chaves utilizadas: "Biological Degradation", "Biopolymers", "Biodegradable Polymers" e "Sustainability", com limitação temporal entre o período de 2002 a 2021. O refinamento ocorreu mediante avaliação dos autores, dado que o objeto de interesse principal é a degradação biológica de materiais poliméricos, considerando as novas tendências sustentáveis que são os polímeros biodegradáveis naturais e sintéticos, levando em consideração as suas aplicações.

\section{Resultados e Discussão}

Os polímeros biodegradáveis feitos de biopolímeros (feitos na natureza) ou de polímeros de base biológica (feitos em uma indústria) estão se tornando cada vez mais importantes na substituição dos polímeros convencionais de bases fósseis não degradáveis que foram descartados nas últimas décadas (Polman et al., 2021). A partir do estudo realizado, foi possível identificar várias aplicações para os polímeros biodegradáveis (naturais e sintéticos) que são as novas tendências sustentáveis.

\subsection{Polímeros Biodegradáveis Naturais}

\subsubsection{Celulose}

A celulose é um polímero natural, renovável, biodegradável e biocompatível (Salama, 2019; Alavi e Nokhodchi, 2019). A celulose é isolada a partir das paredes das células na forma de microfibras por meio de extração química. A sua estrutura química é apresentada na Figura 2.

Figura 2: Estrutura química da celulose.

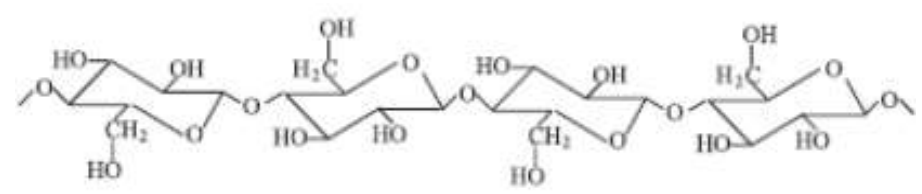

Fonte: Klemm et al. (2005).

Alavi e Nokhodchi (2019) avaliaram as propriedades antimicrobianas e cicatrizantes de nanobiofilmes de óxido de zinco $(\mathrm{ZnO})$, hidrogéis e bionanocompósitos à base de celulose, polímeros de quitosana e alginato. Os autores concluíram que o acoplamento de nanomateriais é uma maneira de aumentar as propriedades mecânicas e antibacterianas dos tecidos que curam enfermidades. 
A celulose não pode ser processada como termoplástico devido a sua infusibilidade, para a obtenção de fibras e filmes, a celulose precisa ser modificada. Há alguns fungos que secretam enzimas que catalisam reações de oxidação da celulose e de seus oligômeros gerados por sua hidrólise. A hidrólise enzimática da celulose pode ser vista na Figura 3. E quando a celulose é exposta a microrganismos na presença de oxigênio, o produto gerado final da biodegradação é o dióxido de carbono e a água (Fechini, 2013; Shankaran, 2018).

Figura 3: Hidrólise enzimática da celulose.

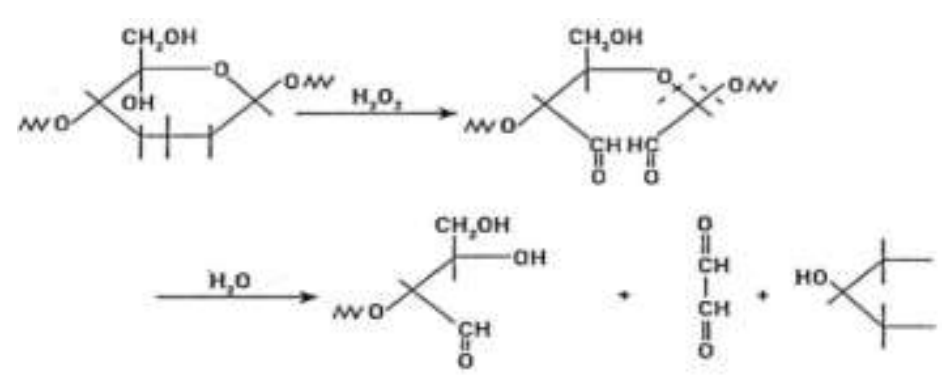

Fonte: Fechini (2013).

Salama (2019) estudou os recentes ensaios para a preparação de materiais híbridos de celulose/fosfato de cálcio, oferecendo perspectivas de desenvolvimento e muitas aplicações novas em diversos campos. Além disso, diferentes formas de celulose e derivados de celulose foram exploradas, como cargas ou modelos para o design de novos materiais funcionais e suporte para o carregamento de diferentes fases de fosfato de cálcio. O autor concluiu que o uso de materiais celulósicos na construção de novos híbridos é muito promissor para aplicações biomédicas e ambientais.

Tong et al. (2020) estudaram o desenvolvimento de filme de celulose altamente transparente, pouco hidrofílico e biodegradável para dispositivos eletroluminescentes flexíveis. Foi relatada uma nova via para a fabricação de filmes de celulose altamente transparentes, pouco hidrofílicos e biodegradáveis. O filme de celulose adquirido não apenas possui alta transparência, mas também apresenta fraca hidrofilicidade e ainda permanece 3,5 MPa de resistência à tração após imersão por dois dias em água deionizada. Além disso, a meia-vida de degradação do filme de celulose foi de 20 dias, e os filmes de celulose também tem melhor termoestabilidade, excelentes propriedades que sugerem potencial para diversas aplicações.

\subsubsection{Amido}

O amido é um polímero de glicose, e constitui 2 tipos de polissacarídeos, que são a amilose (Figura 4) e a amilopectina, além disso, é encontrado em algumas raízes como a mandioca e batata-doce, além de alguns tipos de caules, a sua biodegrabilidade se deve, aos átomos de oxigênio presentes na cadeia principal e no anel. A amilose é cristalina, apresentase insolúvel em água à temperatura próxima da temperatura de ebulição. A amilopectina é insolúvel nestas mesmas condições apresentadas (Fechini, 2013; Hemamalini \& Dev 2017; Ziegler-Borowska, 2019). 
Figura 4: Estrutura química da amilose.

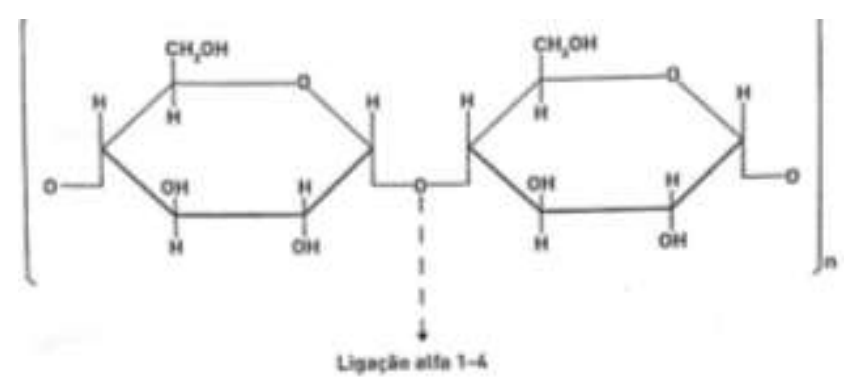

Fonte: Fechini (2013).

Hemamalini e Dev (2017) apresentaram um trabalho que relata tentativas feitas na eletrofiação de amido e suas possíveis aplicações em engenharia biomédica e de tecidos. O amido é um polímero emergente na área de pesquisa biomédica devido à sua facilidade de disponibilidade, baixo custo e valores biológicos. O objetivo da revisão foi fornecer um relatório abrangente sobre a produção de amido na forma fibrosa. Foi estudada a eletrofiação do amido, a eletrofiação de fibras de amido puro e a eletrofiação de amido modificado. Os autores concluíram que o polímero de amido é um dos promissores polissacarídeos que encontra uma ampla aplicação biomédica como em biossensores, revestimentos e curativos.

Tabasum et al. (2018) estudaram o amido de milho com polímeros naturais e sintéticos, e nanopartículas inorgânicas com modelagem matemática. Os métodos mais utilizados para modificar o amido são modificações físicas, químicas, enzimáticas e genéticas. Foi avaliada a combinação de amido com alguns polímeros naturais como: a celulose, a quitosana, a gelatina, entre outros. Já a combinação com polímeros sintéticos do amido de milho com: álcool polivinílico, poliacrilato, entre outros. Os autores concluíram que o amido de milho pode ser facilmente modificado usando diferentes métodos físicos ou químicos, que são utilizados para combinar amido com polímeros naturais e sintéticos. Essas misturas de amido apresentaram uma biocompatibilidade apropriada, propriedades físicas e taxa de degradação satisfatória.

Ziegler-Borowska (2019) detalhou a síntese de novas MNPs (nanopartículas magnéticas) funcionais revestidos com amido através de um método simples, rápido e eficiente de funcionalização de superfície. Com base em pesquisas anteriores na área de síntese de MNPs e aplicações biomédicas, foi demonstrada imobilização eficaz de HSA (albumina humana) para essas nanopartículas sem perda de atividade proteica. Os materiais foram caracterizados por espectroscopia de reflexão total atenuada no infravermelho com transformada de Fourier (ATR-FTIR), microscopia eletrônica de varredura (MEV) e transmissão (MET), espalhamento dinâmico de luz (DLS) e difração de raios-X (DRX). O método permitiu a modificação de polissacarídeos e nanopartículas em relação a materiais enriquecidos com aminoácidos. Pode-se esperar que este método de aminação rápida sem solvente encontre aplicação na química de materiais e polímeros. Os autores concluíram que a eficiência da imobilização da albumina nas nanopartículas revestidas com amido foi muito alta.

\subsubsection{Quitina e Quitosana}

Os polímeros de quitina e quitosana são aminopolissacarídeos naturais com estruturas únicas, propriedades multidimensionais, funções altamente sofisticadas aplicações abrangentes em aplicações biomédicas e outras áreas industriais. A quitosana apresenta uma gama de aplicações na indústria farmacêutica, em cosméticos, membranas poliméricas, biomateriais, entre outros. É bastante atrativo, pois é um material obtido de uma fonte renovável e biodegradável. Quitina e a quitosana possuem imensas possibilidades de modificações químicas e mecânicas para gerar novas propriedades, funções e aplicações especialmente na área biomédica. Apesar de sua enorme disponibilidade, a utilização de quitina tem sido restrito por sua insolubilidade. A quitina possui características de biocompatibilidade, biodegradabilidade e não toxicidade e atividade 
antimicrobiana (Pillai, Paul e Sharma, 2009). A desacetilição da quitina é parcialmente desacetilado para a obtenção da quitosana, podendo ser visualizada na Figura 5.

Figura 5: Desacetilação da quitina para obter a quitosana.

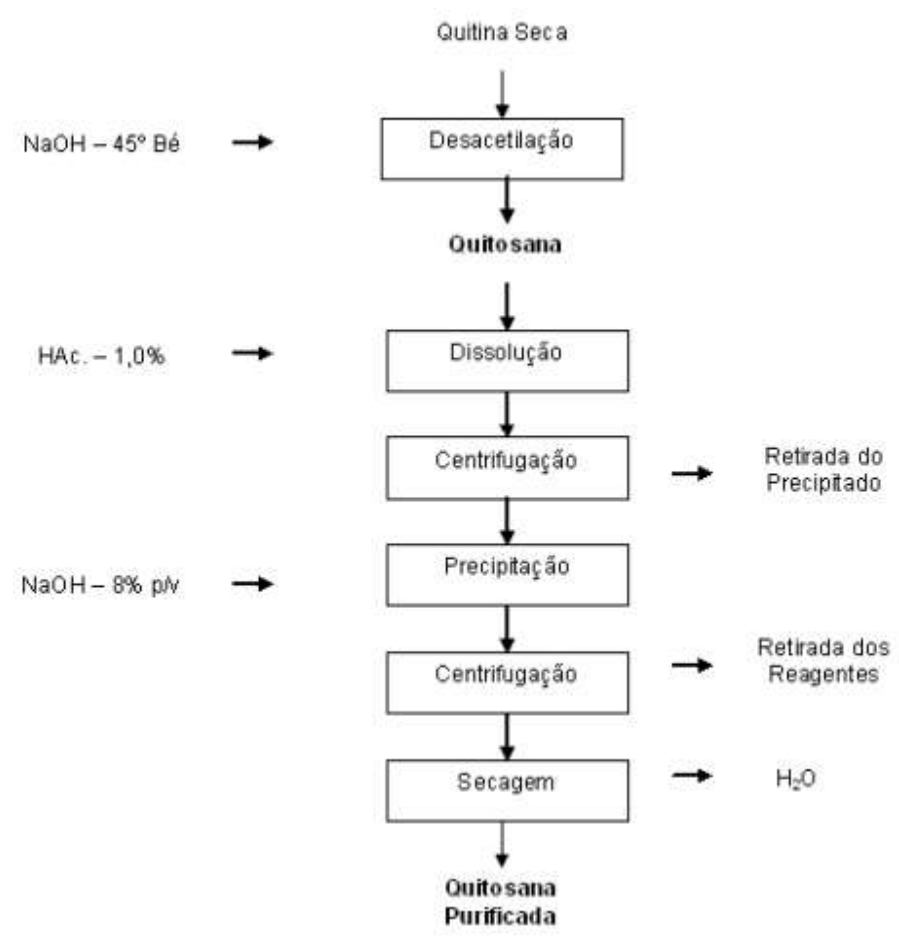

Fonte: Moura et al. (2006).

Pillai, Paul e Sharma (2009) obtiveram uma revisão que discute as várias tentativas para solucionar o problema do ponto de vista da química e da estrutura da quitina e quitosana, destacando as desvantagens e vantagens de cada método apresentado. Foram abordadas as estruturas de quitina e quitosana, modificações químicas, critérios para solubilidade do polímero, a quitina e solubilidade de quitosana, dissolução por produtos químicos inorgânicos, e dissolução da quitina por ácidos fortes e solventes polares. Os autores concluíram que estes são biopolímeros com imensa possibilidade estruturais para modificações químicas e mecânicas para gerar novas propriedades, funções e aplicações especialmente na área biomédica.

Hai e Sugimoto (2018) apresentaram uma modificação superficial de quitina e quitosana com poli (3-hexiltiofeno) por polimerização oxidativas. Foram realizadas modificações bem-sucedidas de biomateriais como a quitina e a quitosana. Além disso, foram preparadas por enxertia direta de poli(3-hexiltiofeno) (P3HT) em suas superfícies usando simples polimerização com cloreto de ferro $\left(\mathrm{FeCl}_{3}\right)$. A estabilidade térmica e a cristalinidade da quitina e quitosana enxertadas mudaram ao enxertar com P3HT. A introdução de P3HT na superfície da quitina e da quitosana melhorou as suas propriedades elétricas e condutividade. Os autores concluíram que a modificação da superfície da quitina e da quitosana foi enxertado com sucesso, usando o método oxidante de polimerização, introduzindo a camada P3HT na superfície ajudando a aumentar a condutividade.

\subsubsection{Polipeptídeos}

As proteínas utilizadas com materiais, muitas não são solúveis e nem passivas de fusão, e são usadas na forma como são encontradas na natureza, como por exemplo, o colágeno e a seda. A degradação das proteínas é um processo que ocorre por meio de reações de hidrólise no agrupamento amida (Fechini, 2013). 
Os polipeptídeos derivados de $\alpha$-aminoácidos que ocorrem naturalmente surgiram como uma família única e versátil de biomateriais bioinspirados que podem ser feitos sob medida para diversas aplicações biomédicas, como liberação controlada de medicamentos, entrega de genes, engenharia de tecidos e medicina regenerativa (Deng et al., 2014). A conformação beta das proteínas da cadeia polipeptídica se estende em uma estrutura em zigue-zague, chamada de folha $\beta$ (Franchetti \& Marconato, 2006). Na Figura 6 podemos observar a conformação beta das proteínas da cadeia polipeptídica.

Figura 6: Conformação beta das cadeias polipeptídicas.

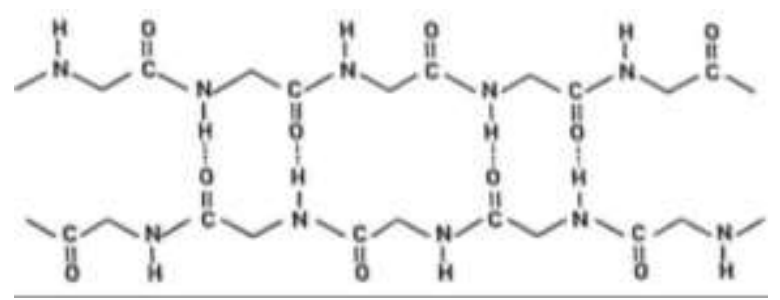

Fonte: Fechini (2013).

\subsubsection{Poliésteres Bacterianos}

Os poliésteres bacterianos naturais são obtidos por uma grande variedade de bactérias via armazenamento intracelular. Estes polímeros são degradáveis, suscetíveis a processamento via fusão e apresenta propriedades desde polímeros rígidos e flexíveis. Dependendo do tipo de suprimento que se dá a bactéria é possível obter vários tipos de poli(hidroxialcanoatos) (PHA) (Fechini, 2013; Sabbagh \& Muhamad, 2017).

A família dos PHA tem um papel muito importante no setor de desenvolvimento de polímeros biodegradáveis no Brasil. O poli(ß-hidroxibutirato) (PHB) é um poliéster sintetizado por microorganismos que ocorrem no solo, este poliéster também pode ser sintetizado quimicamente pela polimerização através de abertura de cadeia $\beta$-butirolactona na presença de catalisadores de alumínio e zinco (Fechini, 2013; Sabbagh \& Muhamad, 2017). O PHB é altamente cristalino, e também é considerado um polímero $100 \%$ biodegradável, que pode ser processado como termoplástico e é resistente a água. Além disso, o PHB possui biodegrabilidade e biocompatibilidade, mas a substituição dos polímeros convencionais por este material não é atrativo devido o seu alto custo (Coutinho et al., 2004). O PHB pode ser visualizada na Figura 7.

Figura 7: Estrutura química do PHB.

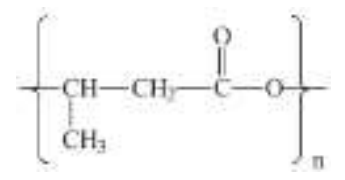

Fonte: Rosa et al. (2002).

\subsection{Polímeros Biodegradáveis Sintéticos}

\subsubsection{Poli( $\varepsilon$-caprolactona) (PCL)}

O poli(E-caprolactona) (PCL) é um poliéster sintético semicristalino obtido a partir da polimerização via abertura de anel da E-caprolactona (Figura 8). O PCL é um polímero bioabsorvível, e tem aplicação na área de reparo de ossos e cartilagens. O PCL possui poucas aplicações devido a sua degradação e reabsorção serem lentas, pois apresenta alto grau de cristalinidade e tem caráter hidrofóbico (Asadian et al., 2019). O PCL é um polímero biodegradável que é usado em aplicações de engenharia de tecidos, graças às suas muitas características favoráveis. No entanto, as superfícies de PCL são conhecidas como hidrofóbicas, levando a uma falta de resposta celular favorável (Amokrane et al., 2018). 
Figura 8: Síntese da poli(ع-caprolactona) via abertura de anel.

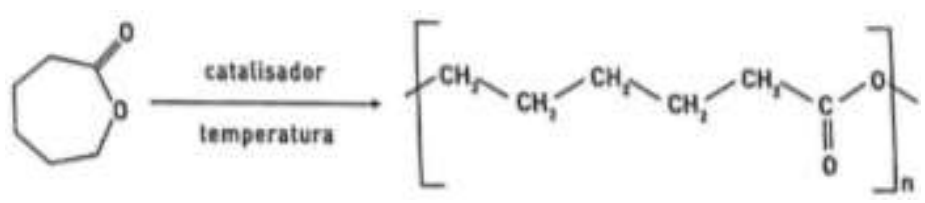

Fonte: Fechini (2013).

Amokrane et al. (2018) apresentaram estudos com enxerto de polímeros bioativos em biomateriais pode melhorar a resposta celular e antibacteriana. O objetivo deste trabalho foi funcionalizar superfícies de PCL através do enxerto de um polímero bioativo. Realizou-se o enxerto de um polímero iônico poli (estireno sulfonato de sódio) (poliNaSS), utilizando irradiação UV em superfícies de PCL em um processo de reação em duas etapas. Vários parâmetros como o tempo de polimerização, o efeito da ativação da superfície, a potência da lâmpada e a concentração de monômeros foram investigadas para otimizar o rendimento do enxerto de poliNaSS. Várias técnicas mostraram que o enxerto de poliNaSS de polímero iônico contendo grupos sulfonato foi bem-sucedido usando radicais de (hidro) peróxidos capazes de iniciar a polimerização de monômeros iônicos em superfícies de PCL.

Asadian et al. (2019) avaliaram mantas nanofibrosas de policaprolactona (PCL) que foram fabricadas por eletrofiação e expostas a um plasma de baixa pressão. A caracterização da superfície foi realizada utilizando várias técnicas: espectroscopia de fotoelétrons de raios X (XPS) para análises químicas de superfície, medições de ângulo de contato com a água (WCA) para exame de molhabilidade, microscopia eletrônica de varredura (MEV) para análise morfológica, caracterização e microscopia de força atômica (AFM) para visualização da topografia de nanofibras individuais antes e após a polimerização plasmática. Além disso, a biocompatibilidade das nanofibras não tratadas e modificadas por plasma também foi avaliada através da propagação de células-tronco da medula óssea. Os resultados obtidos revelaram que a exposição plasmática afetou a morfologia. Os autores concluíram que a incorporação de tiol via polimerização por plasma pode afetar positivamente a resposta celular de malhas nanofibrosas e, portanto, ter grande potencial em aplicações de engenharia de tecidos.

\subsubsection{Poli(ácido láctico) (PLA)}

O poli (ácido lático) (PLA) é um tipo de polímero que foi amplamente estudado devido a propriedades excepcionais como biocompatibilidade, biodegradabilidade e alta resistência (Nordin et al., 2019). O PLA é produzido pela polimerização via abertura de anel lactídeos dimérico, dependendo do lactídeo, o PLA produzido será altamente cristalino ou completamente amorfo. O motivo da sua alta cristalinidade é a estereorregularidade dos lactídeos e, o PLA amorfo ocorre devido à falta desta estereorregularidade. Entre os biopolímeros convencionais, o PLA é uma alternativa promissora, porque o monômero de PLAlactídeo é extraído de fontes naturais (Dubey et al., 2017).

Nordin et al. (2019) relataram o desenvolvimento de compósitos poliméricos condutores a partir de Poli(ácido láctico)PLA/Poliuretano termoplástico (TPU) e misturas preenchidas com nanoplacas de grafeno. O PLA possui algumas boas propriedades, como biocompatibilidade, biodegradabilidade e alta resistência, porém a fragilidade tornou-se a desvantagem deste polímero para ser utilizado em campos mais amplos. O TPU possui alta resistência e tenacidade com boa biocompatibilidade, o que pode melhorar a desvantagem do PLA. A mistura de PLA/TPU foi preparada pelo processo de mistura por fusão com a adição de nanoplacas de grafeno $(\mathrm{GnP})$. Os autores concluíram que a presença de GnP afetou algumas propriedades do compósito, como resistência elétrica, morfológica e à tração. 
Dentre as aplicações do PLA, incluem-se as áreas médica, como por exemplo, parafusos, implantes bioabsorsíveis, entre outras aplicações em outras áreas, com exemplo como substituinte do PET nas embalagens para bebidas (Fechini, 2013). A Figura 9 apresenta a polimerização do PLA.

Figura 9: Planos de polimerização do PLA.

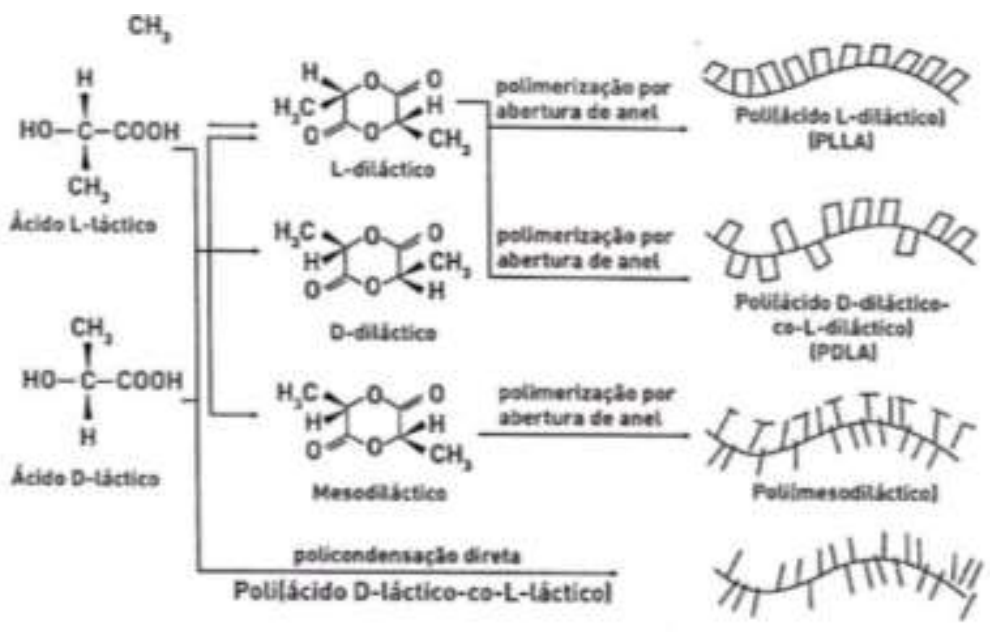

Fonte: Fechini (2013).

\subsubsection{Poli(ácido glicólico) (PGA)}

O poli(ácido glicólico) (PGA), Figura 10 é um polímero bastante utilizado, pois possui alta temperatura de fusão cristalina $\left(-230^{\circ} \mathrm{C}\right)$, alta cristalinidade e baixa solubilidade, possuindo aplicações na área médica (Fechini, 2013).

Figura 10: Estrutura química do PGA.

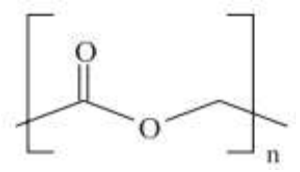

Fonte: Barbanti, Zavaglia e Duek (2005).

Jem e Tan (2020) estudaram o PGA que possui estrutura semelhante ao PLA, apresentando características promissoras como uma boa biodegradabilidade e propriedades de barreira, o que é potencialmente um suplemento benéfico ao PLA. O PGA e sua combinação com o PLA foram amplamente estudados em aplicações biomédicas, devido os custos de produção ainda ser relativamente alto. O desenvolvimento de novas tecnologias de produção e o advento de regulamentações governamentais são os principais impulsionadores da transição global para os polímeros biodegradáveis. Recentemente, vários regulamentos governamentais foram lançados que restringem o uso de polímeros tradicionais e facilitam as aplicações dos biodegradáveis. O PGA pode ser derivado de gases residuais industriais usando uma tecnologia de produção inovadora, que reduz as emissões de carbono e seu custo de produção. Ao desenvolver a tecnologia de produção e composição, o PGA pode ser combinado com o PLA para desempenhar um papel essencial na indústria de plástico sustentável e ecologicamente correta, especialmente para produtos de uso único que requerem degradação rápida em temperatura ambiente ou no ambiente natural. 


\subsubsection{Poli(butileno succinato) (PBS)}

O poli(butileno succinato) (PBS) ilustrado na Figura 11 possui a biodegrabilidade, que quando na forma de filmes, possui propriedades mecânicas similares as do polietileno de baixa densidade. A partir da incorporação de ácido adípico na síntese do PBS, se obtém o copolímero poli(butileno succinato-co-butileno adipato) (PBSA). Este copolímero PBSA possui uma maior biodegrabilidade devido possuir menor cristalinidade (Fechini, 2013).

Figura 11: Estrutura química do PBS.

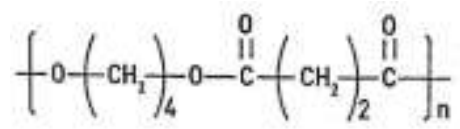

Fonte: Fechini (2013).

Zhao et al. (2020) avaliaram um biocompósito de PBS totalmente biodegradável fabricado a partir de fibra de PBS ultrafina e resíduos de papel (RP) usando um método de fabricação de papel e moldagem por compressão. Um biocompósito de PBS contendo 60\% em peso de RP apresentou resistência à tração, alongamento na ruptura, resistência à flexão, módulo de flexão, resistência ao cisalhamento interlaminar e resistência ao impacto, superiores aos dos biocompósitos de PBS relatados anteriormente. A mecânica dos danos revelou que a tremenda deformação das fibras vegetais (FV) do RP e sua rebitagem melhoraram as propriedades mecânicas dos biocompósitos RP/PBS. A razão de perda de peso do biocompósito RP/PBS-60\% em peso durante a biodegradação atingiu 95,1\% em peso após imersão em uma solução de enzima por 35 dias. Esta abordagem de fabricação forneceu uma estratégia nova e fácil para desenvolver biocompósitos de PBS de alto desempenho, de baixo custo e totalmente biodegradáveis.

\subsubsection{Poli(álcool vinílico) (PVA) e Poli(N-vinil-2-pirrolidona) (PVP)}

O poli(álcool vinílico) (PVA) é polímero semicristalino, solúvel em água, exibe uma baixa condutividade elétrica e é considerado biodegradável (Pravakar et al., 2019). Outro exemplo de polímero solúvel em água é o poli(N-vinil-2-pirrolidona) (PVP). A seguir na Figura 12 é apresentado as estrutura químicas do PVA e do PVP.

Figura 12: Estrutura química: (a) PVA e (b) PVP.

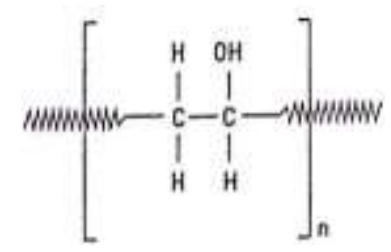

(a)

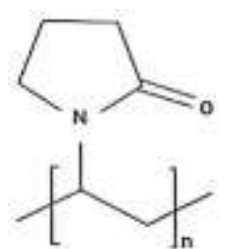

(b)

Fonte: Fechini (2013).

Teodorescu, Bercea e Morariu (2019) discutiram as tendências futuras para onde a pesquisa de biomateriais está se dirigindo, mostrando as oportunidades promissoras que podem oferecer o PVA e o PVP. O PVA atraiu considerável interesse de pesquisa e é reconhecido entre o maior volume de polímeros sintéticos que foram produzidos mundialmente por quase um século. Isso se deve às suas propriedades excepcionais que ditaram seu uso extensivo em uma ampla variedade de aplicações, especialmente nas áreas médica e farmacêutica. Contudo, estudos revelaram que os biomateriais à base de PVA apresentam 
algumas limitações que podem restringir seu uso ou performance. Para superar essas limitações, vários métodos foram relatados, entre os quais a mistura com PVP apresentou resultados promissores. Portanto, foi constatado o efeito sinérgico das misturas entre PVA e PVP, variando a preparação e propriedades, podendo ser aplicados em biomateriais.

\subsection{Aplicações dos Polímeros Biodegradáveis}

Diante do exposto, foi realizado um resumo dos principais artigos pesquisados neste trabalho por meio da Tabela 2, apresentando os autores, o polímero biodegradável, bem como as suas principais aplicações e/ou melhoria de propriedades.

Tabela 2: Aplicação e melhoria de propriedades de alguns polímeros biodegradáveis.

\begin{tabular}{|c|c|c|}
\hline Autores & Polímeros Biodegradáveis & Aplicações e/ou melhoria de propriedades \\
\hline Hai e Sugimoto (2017) & Quitina e quitosana & Melhoria de condutividade \\
\hline Hermamalini e Dev (2017) & Amido & Biossensores, revestimentos e curativos \\
\hline Tabasum et al. (2018) & Amido & $\begin{array}{c}\text { Biocompatibilidade, propriedades físicas e taxa } \\
\text { de degradação apropriada }\end{array}$ \\
\hline Alavi e Nokhodchi (2019) & Celulose, quitosana & Propriedades mecânicas e antibacterianas \\
\hline Asadian et al. (2019) & PCL & Engenharia de tecidos \\
\hline Nordin et al. (2019) & PLA & $\begin{array}{l}\text { Aumento da resistência à tração e elétrica, } \\
\text { melhoria das propriedades morfológicas }\end{array}$ \\
\hline Salama (2019) & Celulose/fosfato de cálcio & Biomédicas e ambientais \\
\hline Teodorescu, Bercea e Morariu (2019) & PVA e PVP & Biomateriais e farmacêutica \\
\hline Jem e Tan (2020) & PGA & Biodegradabilidade e propriedades de barreira \\
\hline Tong et al. (2020) & Celulose transparente & Termoestabilidade e ambientais \\
\hline Zhao et al. (2020) & PBS & Biocompósitos de baixo custo e biodegradáveis \\
\hline
\end{tabular}

Fonte: Autores.

\section{Considerações Finais}

A utilização dos polímeros biodegradáveis (naturais e sintéticos) surge como uma opção para ampliar a solução dos problemas decorrentes da poluição ambiental, devido à quantidade significativa gerada de materiais poliméricos que não são facilmente degradados. Portanto, a aplicação dos polímeros biodegradáveis vem como uma tendência para a preservação ambiental por apresentarem uma taxa de degradação apropriada. Além desses aspectos abordados, o esgotamento do petróleo para obtenção dos polímeros sintéticos, implica na busca de pesquisas por novas alternativas de materiais sustentáveis. De maneira geral, ficou constatado que o estudo da modificação dos polímeros biodegradáveis naturais como a celulose, amido, quitina, quitosana, polipeptídeos e poliésteres bacterianos resultou na melhoria das propriedades mecânicas, antibacterianas, termoestabilidade, biocompatibilidade e não toxicidade para serem aplicados como biomateriais. Já as alterações realizadas nos polímeros biodegradáveis sintéticos como a poli(e-caprolactona), poli(ácido láctico), poli(ácido glicólico), poli(butileno succinato), poli(álcool vinílico) e poli(N-vinil-2-pirrolidona) resultou na melhoria de propriedades para serem utilizados na engenharia de tecidos como em implantes bioabsorsíveis, além de serem direcionados também para embalagens de bebidas.

\section{Agradecimentos}

Os autores agradecem a FAPESB, ao CNPq e a CAPES pelas bolsas de pesquisa concedidas.

\section{Referências}

Alavi, M., \& Nokhodchi, A. (2019). An Overview on Antimicrobial and Wound Healing Properties of Zno Nanobiofilms, Hydrogels, and Bionanocomposites Based on Cellulose, Chitosan, and Alginate Polymers. Carbohydrate Polymers, 227, 2-6. https://doi.org/10.1016/j.carbpol.2019.11534 9

Amokrane, G., Falentin-Daudré, C., Ramtani, S., \& Migonney, V. (2018). A Simple Method to Functionalize PCL Surface by Grafting Bioactive Polymers Using UV Irradiation. IRBM, 39, 268-278. https://doi.org/10.1016/j.irbm.2018.07. 002 
Asadian, M., Onyshchenko, I., Thiry, D., Cools, P., Declercq, H., Snyders, R., Morent, R., \& Geyter, N. D. (2019). Thiolation of Polycaprolactone (PCL) Nanofibers by Inductively Coupled Plasma (ICP) Polymerization: Physical, Chemical and Biological Properties. Applied Surface Science, 479, 942-952. https://doi. org/10.1016/j.apsusc.2019.02.178

Ashton, E. G., Jr, W. K., Demori, R., Candido, L. H. A., \& Mauler, R. (2016). Recycling Polymeric Multi-Material Products Through Micronization. Journal of Cleaner Production, 116, 268-278. https://doi.org/10.1016/ j.jclepro.2016.01.018

Barbanti, S. H., Zavaglia, C. A. C., \& Duek, E. A. R. (2005). Polímeros Bioreabsorvíveis na Engenharia de Tecidos. Polímeros: Ciência e Tecnologia, 15(1), 13-21. https://doi.org/10.1590/S0104-14282005000100006

Bilal, M., \& Iqbal, H. M. N. (2019). Naturally-Derived Biopolymers: Potential Platforms for Enzyme Immobilization. International Journal of Biological Macromolecules, 130, 462-482. https://doi.org/10.1016/j.ijbiomac.2019.02.152

Boonmee, C. (2016). Degradation of poly(lactic acid) under simulated landfill conditions. Environment and Natural Resources Journal, 14(2), 1-9. https://doi.org/10.14456/ ennrj.2016.8.

Braido, R. S., Borges, L. E. P., \& Pinto, J. C. (2018). Chemical Recycling of Crosslinked Poly(Methyl Methacrylate) and Characterization of Polymers Produced with the Recycled Monomer. Journal of Analytical and Applied Pyrolysis, 132, 47-55. https://doi.org/10.1016/j.jaap.2018.03.01 7

Brito, G. F., Agrawal, P., Araújo, E. M., \& Mélo, T. J. A. (2011). Biopolímeros, Polímeros Biodegradáveis e Polímeros Verdes. Revista Eletrônica de Materiais e Processos (REMAP), 6(2), 127-139. http://www2.ufcg.edu.br/revista-remap/index.php/REMAP/article/view/222/204

Coutinho, B. C., Miranda, G. B., Sampaio, G. R., Souza, L. B. S., Santana, W. J., \& Coutinho, H. D. M. (2004). A importância e as vantagens do polihidroxibutirato (plástico biodegradável). Holos, 20, 76-81. https://doi.org/10.15628/holos.2004.49

Deng, C., Wu, J., Cheng, R., Meng, F., Klok, H-A., \& Zhong, Z. (2014). Functional Polypeptide and Hybrid Materials: Precision Synthesis Via Amino Acid N-Carboxyanhydride Polymerization and Emerging Biomedical Applications. Progress in Polymer Science, 39, 330-364. https://doi.org/10.1016/j.progpolymsci.2013.10.008

Dias, J. C. (2016). Rotas de Destinação dos Resíduos Plásticos e seus Aspectos Ambientais: Uma Análise da Potencialidade da Biodegradação. Dissertação de Mestrado em Planejamento Energético/COPPE, Universidade Federal do Rio de Janeiro, Rio de Janeiro.

Dubey, S. P., Thakur, V. K., Krishnaswamy, S., Abhyankar, H. A., Marchante, V., \& Brighton, J. L. (2017). Progress in Environmental-Friendly Polymer Nanocomposite Material from PLA: Synthesis, Processing and Applications. Vacuum, 146, 655-663. https://doi.org/ 10.1016/j .vacuum.2017.07.009

Farias, S. S., Siqueira, S. M. C., Cristino, J. H. S., \& Rocha, J. M. (2016). Biopolímeros: Uma Alternativa para Promoção do Desenvolvimento Sustentável. Revista Geonorte, 7(26), 61-77. https://periodicos.ufam.edu.br/index.php/revista-geonorte/article/view/2759/2495

Fechini, G. J. M. (2013). Polímeros Biodegradáveis: Tipos, Mecanismos, Normas e Mercado Mundial. Editora Mackenzie, 120p.

Fraga, S. C. L. (2014). Reciclagem de Materiais Plásticos: Aspectos Técnicos, Econômicos, Ambientais e Sociais.: Editora Érica Ltda, 120p.

Franchetti, S. M. M., Marconato, J. C. (2006). Polímeros Biodegradáveis - Uma Solução Parcial para Diminuir a Quantidade dos Resíduos Plásticos. Química Nova, 29(4), 811-816. https://www.scielo.br/pdf/qn/v29n4/30263.pdf

Gomes, J. (2010). Poluição Atmosférica: Um Manual Universitário. Pubindustria, 266p.

Hai, T. A. P., \& Sugimoto, R. (2018). Surface modification of chitin and chitosan with poly(3-hexylthiophene) via oxidative polymerization. Applied Surface Science, 434, 188-197. https://doi.org/10.1016/j.apsusc .2017.10.197

Hamad, K., Kaseem, M., \& Deri, F. (2013). Recycling of Waste From Polymer Materials: An Overview of the Recent Works. Polymer Degradation And Stability, 98, 2801-2812. https://doi.org/10. 1016/j.polymdegradstab.2013.09.025

Hatti-Kaul, R., Nilsson, L. J., Zhang, B., Rehnberg, N., \& Lundmark, S. (2019). Designing Biobased Recyclable Polymers for Plastics. Trends in Biotechnology, 38(1), 50-67. https://doi.org/10.1016/j.tibtech.2019.04.011

Hemamalini, T., \& Dev, V. R. G. (2017). Comprehensive review on electrospinning of starch polymer for biomedical applications. International Journal of Biological Macromolecules, 106, 712-718. https://doi.org/10.1016/j.ijbiomac.2017.08.0 79

Jim, K. J., \& Tan, B. (2020). The development and challenges of poly (lactic acid) and poly(glycolic acid). Advanced Industrial and Engineering Polymer Research, 3, 60-70. https://doi.org/10.1016/j.aiepr.2020.01.002

Klemm, D., Heublein, B., Fink, H. P., \& Bohn, A. (2005). Cellulose: Fascinating Biopolymer and Sustainable Raw Material. Angewandte Chemie International Edition, 44, 3358-3393. https://doi.org/10.1002/anie.200460587

Landim, A. P. M., Bernardo, C. O., Martins, I. B. A., Francisco, M. R., Santos, M. B., \& Melo, N. R. D. (2016). Sustentabilidade quanto às Embalagens de Alimentos no Brasil. Polímeros, 26, 82-92. https://doi.org/10.1590/0104-1428.1897

Lendlein, A., \& Sisson, A. (2011). Handbook of Biodegradable Polymers: Synthesis, Characterization and Applications. Wiley-VCH, 426p.

Lima, E. G., \& Okimoto, M. L. L. R. (2009). Revisão da aplicação de produtos biopolímeros obtidos pela reciclagem de plásticos em design. Revista Iberoamericana de Polímeros, 10(5), 244-259. http://www.ehu.eus/reviberpol/pdf/SE P09/lima.pdf

Mano, E. B., Pacheco, É. B. A. V., \& Bonelli C. M. C. (2010). Meio Ambiente Poluição e Reciclagem. 2a Ed., São Paulo: Editora Blucher, 200p. 
Maris, J., Bourdon, S., Brossard, J-M., Cauret, L., Fontaine, L., \& Montembault, V. (2018). Mechanical Recycling: Compatibilization of Mixed Thermoplastic Wastes. Polymer Degradation and Stability, 147, 245-266. https://doi.org /10.1016/j.polymdegradstab.2017.11.001

Moura, C., Muszinski, P., Schmidt, C., Almeida, J., \& Pinto, L. (2006). Quitina e Quitosana Produzidas a Partir de Resíduos de Camarão e Siri: Avaliação do Processo em Escala Piloto. Vetor, 16, 37-45. http://repositorio.furg.br/ handle/1/4604

Niaounakis, M. (2019). Recycling of Biopolymers - The Patent Perspective. European Polymer Journal, 114, 464-475. https://doi.org/10.1016/ j.eurpolymj.2019.02.027

Nordin, N. M., Buys, Y. F., Anuar, H., Ani, M. H., \& Pang, M. M. (2018). Development of Conductive Polymer Composites from PLA/TPU Blends Filled with Graphene Nanoplatelets. Materials Today: Proceedings, 17, 500-507. https:// doi.org/10.1016/j.matpr.2019.06.328

Pillai, C. K. S., Paul, W., \& Sharma, C. P. (2009). Chitin and Chitosan Polymers: Chemistry, Solubility and Fiber Formation. Progress in Polymer Science, 34, 641-678. https://doi.org/10.1016/j.prog polymsci.2009.04.001

Piva, A. M., \& Wiebeck, H. (2004). Reciclagem do Plástico: Como Fazer da Reciclagem um Negócio Lucrativo. Artliber Editora, 119p.

Polman, E. M. N., Gruter, G. J. M., Parsons, J. R., Tietema, A. (2021). Comparison of the aerobic biodegradation of biopolymers and the corresponding bioplastics: A review. Science of the Total Environment, 753, 1-13. https:// doi.org/10.1016/j.scitotenv.2020.141953

Pradella, J. G. C. (2006). Biopolímeros e Intermediários Químicos. Relatório Técnico $n^{\circ} 84$ 396-205. Centro de Tecnologia de Processos e Produtos Laboratório de Biotecnologia Industrial- LBI/CTPP, São Paulo.

Pravakar, O., Siddaiah, T., Ramacharyulu, P. V. R. K., Gopal, N. O., Ramu, C., Nagabhushana, H. (2019). Spectroscopic, thermal, structural and electrical studies on $\mathrm{VO}^{2+}$ ions doped PVA/MAA: EA polymer blend films. Journal of Science: Advanced Materials and Devices, 4, 267-275. https://doi.org/ 10.1016/j.jsamd.2019.03.004

Rosa, D. S., Chui, Q. S. H., Filho, R. P., Agnelli, J. A. M. (2002). Avaliação da Biodegradação de Poli- $\beta$-(Hidroxibutirato), Poli- $\beta$-(Hidroxibutirato-covalerato) e Poli-E-(caprolactona) em Solo Compostado. Polímeros: Ciência e Tecnologia, 12( 4), 311-317. https://doi.org/10.1590/S0104-1428200 20 00400015

Rosa, D. S., Pantano Filho, R. (2003). Biodegradação: Um Ensaio com Polímeros, São Paulo: Editora Moara, 146p.

Sabbagh, F., Muhamad, I. I. (2017). Production of poly-hydroxyalkanoate as secondary metabolite with main focus on sustainable energy. Renewable and Sustainable Energy Reviews, 72, 95-104. https://doi.org/10.1016/j.rser.2016.11.012

Shankaran, D. R. (2018). Cellulose nanocrystals for health care applications. In: Mohan Bhagyaraj, S.M., Oluwafemi, O.S., Kalarikkal, N., Thomas, S. (Eds.). Micro and Nano Technologies. 415-459. https://doi.org/10.1016/B978-0-08-101971- 9.00015-6

Salama, A. (2019). Cellulose/Calcium Phosphate Hybrids: New Materials for Biomedical and Environmental Applications. International Journal of Biological Macromolecules, 127, 606-617. https://doi.org/10.1016/j.ijbiomac.2019.01.13 0

Smith, R. (2005). Biodegradable Polymers for Industrial Applications. $1^{\text {a }}$ Ed., Press LLC, USA, 548p.

Tabasum, S., Younas, M., Zaeem, M. A., Majeed, I., Majeed , M., Noreen, A., Iqbal , M. N., Zia, K. M. (2018). A Review on Blending of Corn Starch with Natural and Synthetic Polymers, and Inorganic Nanoparticles with Mathematical Modeling. International Journal of Biological Macromolecules, 122, 969996. https: //doi.org/10.1016/j.ijbiomac.2018.10.092

Teodorescu, M., Bercea, M., Morariu, S. (2019). Biomaterials of PVA and PVP in medical and pharmaceutical applications: Perspectives and challenges. Biotechnology Advances, 37(1), 109-131. https://doi.org/10.1016/j.biot echadv.2018.11.008

Thomas, P., Rumjit, N P., Lai, C. W., Johan, M. R. B., Saravanakumar, M. P. (2020). Polymer-Recycling of Bulk Plastics. Encyclopedia of Renewable and Sustainable Materials, 2, 432-454. https://doi.org/ 10.1016/B978-0-12-803581-8.10765-9

Tong, R, Chen, G, Tian, J, He, M. (2020). Highly transparent, weakly hydrophilic and biodegradable cellulose film for flexible electroluminescent devices. Carbohydrate Polymers, 227, 1-8. https://doi.org/10.1016/j.carbpol.2019.11536 6

Valerio, O., Muthuraj, R., Codou, A. (2020). Strategies for polymer to polymer recycling from waste: Current trends and opportunities for improving the circular economy of polymers in South America. Current Opinion in Green and Sustainable Chemistry, 25, 100381. https://doi.org $/ 10.1016 /$ j.cogsc.2020.100381

Vesilind, P. A., Morgan, S. M. (2011). Introdução à Engenharia Ambiental. Tradução da 2a Ed. norte-americana. São Paulo: Cegage Learning, 456p.

Zhao, L., Huang, H., Han, Q., Yu, Q., Lin, P., Huang, S., Yin, X., Yang, F., Zhan, J. Wang, H., Wang, L. (2020). A novel approach to fabricate fully biodegradable poly(butylene succinate) biocomposites using a paper-manufacturing and compression molding method. Composites Part A, 139, 1-9. https://do i.org/10.1016/j.compositesa.2020.106117

Ziegler-Borowska, M. (2019). Magnetic nanoparticles coated with aminated starch for HSA immobilization- simple and fast polymer surface functionalization. International Journal of Biological Macromolecules, 136, 106-114. https:// doi.org/10.1016/j.ijbiomac.2019.06.044 